\title{
A SIMPLE INOCULATION RACK AND MODIFIED TECHNIQUE FOR RAPID SUBCULTURING AND ANTIBIOTIC TESTING
}

\author{
BY \\ L. F. HEWITT \\ From the Serum Research Institute (Medical Research Council), Carshalton, Surrey
}

(RECEIVED FOR PUBLICATION JULY, 1950)

The test-tube rack usually used by bacteriologists to hold culture tubes was inherited from chemical laboratories and has disadvantages for bacteriological work in which the culture tube is held in a sloping position for inoculation. The inoculation rack described here has been in use for some years and has been found to be most efficient and to lend itself to a modified rapid technique for subculturing and for titrating antibiotics.

The tubes are held in a sloping position at an angle of about $45^{\circ}$ to the vertical as shown in the figure. The advantage is that cotton wool plugs (or metal caps)
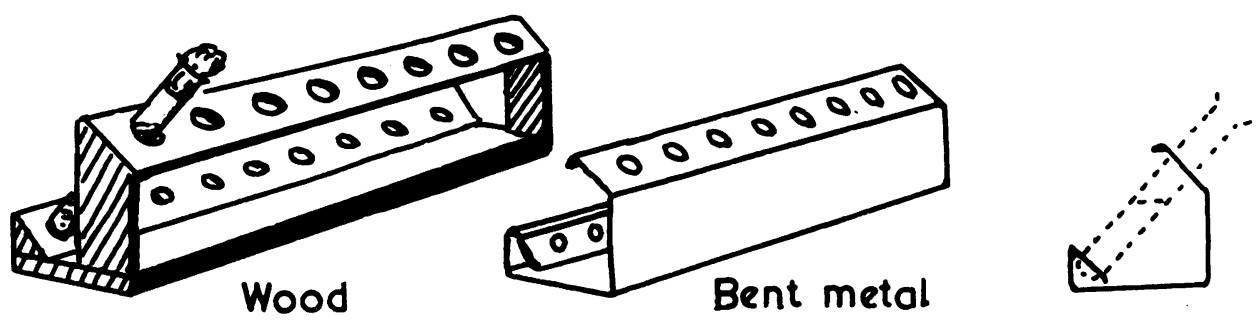

can be removed and pipetting and inoculating carried out while the tubes are still in the rack instead of being held in the hand. The slope prevents particles of contaminated dust falling into the culture medium as they are trapped in the top of the tube, and during incubation particles from the plug do not fall into the medium. The angle of slope of the tubes is the one normally used for inoculation or pipetting, so that if many tubes are to be inoculated or to receive serial dilutions of antibiotic, all the cotton wool plugs are removed and placed on a flamed surface, e.g., the bottom of an upturned wire mesh basket. Pipetting and inoculation can then be carried out at leisure without the risk of contamination from falling dust particles, and it is found in practice that, even when the experiment is interrupted and the tubes are left open for a time before the plugs are replaced, contamination does not occur. Flaming of the tops of tubes before and after inoculation is effective in destroying any contaminating particles.

A further advantage of the sloping racks is in the ease of examining the cultures after incubation. The rack need not be held up for inspection, but the slope of the 
tubes is convenient for direct observation on the bench. For this purpose racks are painted a matt black so that turbidity is easily seen against the dark background of the base of the rack. For biochemical work white-painted racks make colour changes easy to detect without the necessity of holding racks up to the light.

The wide base of the rack makes it practically impossible to overturn the rack either on the bench or incubator no matter how carelessiy it is handled. The construction of the rack is simple and inexpensive and may be of wood, or it may be made from a single piece of bent aluminium alloy or stainless steel.

\section{Summary}

A simple rack is described in which culture tubes are held in a sloping position suitable for inoculating and pipetting operations without the risk of air contamination or the need to hold tubes in the fingers. Besides the greater rapidity achieved in carrying out multiple subcultures and antibiotic tests and the decrease in the contamination of cultures, the inspection of cultures is accelerated and simplified. 